\title{
Investigation of the Local Universe by Means of IRAS Galaxies
}

\author{
A. M. Mickaelian \\ Byurakan Astrophysical Observatory and Isaac Newton Institute \\ Armenian Branch, Byurakan 378433, Armenia
}

\begin{abstract}
The Byurakan-IRAS galaxy (BIG) sample is based on optical identifications of IRAS PSC sources (Beichman, C. A. et al., eds. 1988, Infrared Astronomical Satellite (IRAS) Catalogs and Atlases: The Point Source Catalog, NASA RP-1190, Washington, DC). It makes use of the IR colours, DSS images, and the First Byurakan Spectral Survey (Markarian, B. E. et al. 1989, Commun. Special Astrophys. Obs., 62, 5). All IRAS sources in the region $+61^{\circ}<\delta<90^{\circ}$ at high galactic latitudes $\left(|b|>15^{\circ}\right)$ in an area of $1487 \mathrm{deg}^{2}$ have been revealed up to the limit of the IRAS survey. The BIG sample (Mickaelian, A. M. 2000, Afz, 43, 425 and references therein) consists of 1500 galaxies, including 870 that were previously known. A redshift survey for brighter objects is being carried out with the SAO (Russia) $6 \mathrm{~m}$, Byurakan Observatory $2.6 \mathrm{~m}$, and $\mathrm{Ob}$ servatoire de Haute Provence $1.93 \mathrm{~m}$ telescopes. Redshifts in the range of 0.009-0.173 have been measured. For fainter objects, including 30 empty fields corresponding to sources with IR colors typical of galaxies, deep imaging is being carried out to reveal faint objects and study their morphologies. These objects are candidate ULIRGs. Many are multiple galaxies and small groups. About half of the galaxies are radio sources, and a number are also X-ray sources. The IR luminosity may be due to normal star formation or triggered by interaction or active galactic nuclei (AGNs). The AGNs and interacting/merging systems among the nearest BIG objects are the most interesting cases: they provide understanding of the properties of activity, starburst, and interaction phenomena and their interrelation, thus allowing a study of the physics and evolution of galaxies in the Local Universe.
\end{abstract}

